\title{
Comment HOX choisit sa cible...
}

Les protéines codées par les gènes Hox se lient à l'ADN par l'intermédiaire d'un domaine carboxy-terminal très conservé appelé homéodomaine (pour revue, voir [1]). Ce domaine de 60 acides aminés est constitué d'un bras amino-terminal qui entre en contact avec le petit sillon de la double hélice d'ADN, et de trois hélices $\alpha$, les hélices 2 et 3 formant un motif hélice-bouclehélice qui amène l'hélice 3 en contact avec le grand sillon de la molécule d'ADN. Un second domaine situé en amont de l'homéodomaine caractérise les protéines HOX; ce domaine, appelé motif YPWM* ou hexapeptide, est séparé de l'homéodomaine par une région de taille variable, désorganisée et par conséquent flexible. In vitro, les homéodomaines se fixent sans grande spécificité, sous la forme de monomères, à de courtes séquences d'ADN dont le cœur est TAAT. Ces séquences sont statistiquement très fréquentes dans le génome. Ces données ne rendent pas compte de la très grande spécificité de l'action des protéines HOX in vivo même si l'on peut imaginer une certaine régulation de l'accessibilité des cibles génomiques par la structure de la chromatine. Ainsi, l'hypothèse de l'existence de facteurs agissant de concert avec les protéines HOX a-t-elle été soulevée.

Un gène candidat codant pour un co-facteur des protéines HOX a tout d'abord été identifié chez la drosophile. Il s'agit du gène extradenticle (exd) dont la mutation, bien que provoquant des transformations homéo-

* Y:tyrosine; $P$ : proline; W: tryptophane; $M:$ méthionine.

$\mathrm{m} / \mathrm{s} n^{\circ} 8$-9, vol. 15, août-septembre 99 tiques, n'affecte pas l'expression des gènes Hox. Ce gène participe, entre autres, avec le gène Hox Ultrabithorax, à la régulation du gène réalisateur decapentaplegic $(d p p)$ dans le mésoderme viscéral; les protéines Exd et Ubx se fixent sous la forme d'un hétérodimère sur des séquences cisrégulatrices de $d p p$. Le gène exd est l'homologue des proto-oncogènes humains pre-B cell homeobox ( $p b x 1$, $p b \times 2, p b \times 3)$, impliqués dans des translocations chromosomiques associées à des leucémies, et du gène $c e h-20 \mathrm{de}$ Caenorhabditis elegans. Cette deuxième famille de gènes code également pour des protéines à homéodomaine regroupées sous le terme général de protéines PBC. L'homéodomaine de ces protéines diffère des homéodomaines classiques par la présence de trois acides aminés supplémentaires à l'extrémité carboxy-terminale de l'hélice 1 (homéodomaine de type TALE : three amino-acid loop extension). Cette caractéristique rapproche les protéines $\mathrm{PBC}$ de la protéine à homéodomaine MAT $\alpha 2$ impliquée dans la détermination du type conjugal de la levure Saccharomyces cerevisiae. Les données génétiques et biochimiques obtenues par un grand nombre de laboratoires convergent vers le modèle d'une fixation coopérative des hétérodimères $\mathrm{PBC} / \mathrm{HOX}$ à la séquence consensus 5'TGAT (T/G)NA(T/C)-3', l'homéodomaine de la protéine $\mathrm{PBC}$ se liant à la séquence TGAT et celui de la protéine HOX à la séquence $(\mathrm{T} / \mathrm{G}) \mathrm{NA}(\mathrm{T} / \mathrm{C})$. La base située en position 6 du site de liaison semble jouer un rôle fondamental dans le choix de la protéine HOX participant à l'hétérodimère (figure 1). Enfin, le motif YPWM est nécessaire à la liaison coopérative des deux homéoprotéines à l'ADN.

Afin d'élucider les mécanismes moléculaires qui sous-tendent la liaison coopérative des hétérodimères PBCHOX à l'ADN, deux laboratoires viennent indépendamment d'effectuer l'analyse cristallographique à haute résolution des complexes ternaires Exd-Ubx/ADN de drosophile [2] et Pbx1-HoxB1/ADN humain [3]. Les deux structures sont identiques, ce qui démontre une remarquable conservation de la fonction au cours de l'évolution. Ainsi que les données biochimiques l'avaient suggéré, les homéodomaines HOX et PBC sont placés tête-bêche, de chaque côté de la double hélice d'ADN qu'ils contactent via leur hélice $\alpha 3$ (figure 1). Toujours en accord avec les observations antérieures, l'hélice $\alpha 3$ de la protéine HOX entre en contact avec l'un des nucléotides situés sur le petit sillon, correspondant au nucléotide $6 \mathrm{du}$ site de liaison. Les deux homéodomaines ne se touchent pas. Cependant, un repliement de l'homéodomaine PBC constitué de l'extrémité amino-terminale de l'hélice $\alpha 1$, des trois acides aminés caractéristiques des homéodomaines TALE et de l'extrémité carboxy-terminale de l'hélice $\alpha 3$, permet la formation d'une poche hydrophobe qui reçoit le motif YPWM de la protéine HOX. La cohésion de cette structure est en partie assurée par une liaison hydrogène entre le résidu tryptophane $(\mathrm{W})$ et l'un des trois acides aminés de l'insertion TALE. Cette caractéristique différencie la famille des protéines à homéodomaine $\mathrm{PBC}$ des autres protéines TALE, et notamment de MAT $\alpha 2$, dans lesquelles les 


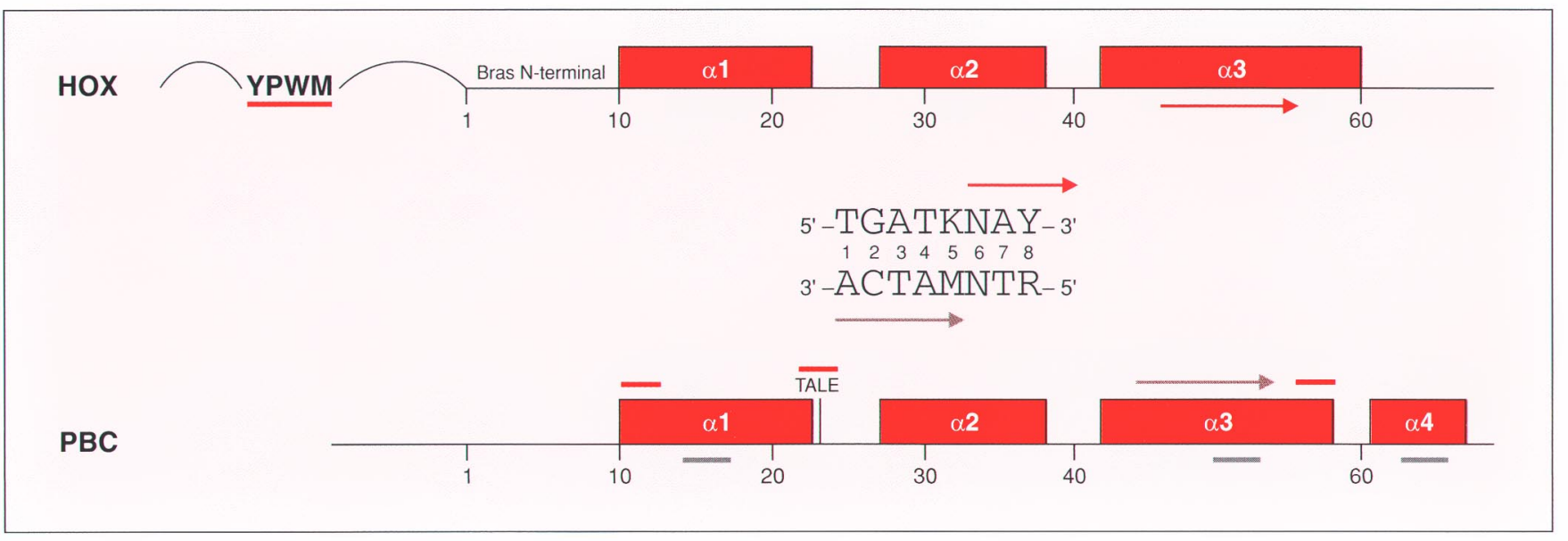

Figure 1. Représentation schématique d'un complexe ternaire PBC-HOX/ADN. Les homéodomaines correspondent aux acides aminés 1 à $60 . \alpha 1$ : hélice $\alpha 1 ; \alpha 2$ : hélice $\alpha 2 ; \alpha 3$ : hélice $\alpha 3 ; \alpha 4$ : hélice $\alpha 4$ de Pbx1. Contacts entre l'hélice $\alpha 3$ de la protéine HOX et l'ADN (flèche rouge). Contacts entre l'hélice $\alpha 3$ de la protéine PBC et l'ADN (flèche grise). Contacts entre l'extrémité amino-terminale de l'hélice $\alpha 1$ de PBC, les 3 acides aminés TALE, l'hélice $\alpha 3$ de PBC et le domaine YPWM de la protéine HOX (trait rouge). Repliement de l'hélice $\alpha 4$ de Pbx1 sur les hélices 1 et 3 (trait gris). $K: G$ ou $T ; Y: T$ ou $C ; M: A$ ou $C ; R: A$ ou $G$.

trois acides aminés TALE ne semblent pas jouer de rôle dans l'interaction avec les partenaires protéiques. Alors que la structure spatiale des deux homéodomaines reste inchangée lors de la formation de l'hétérodimère, celle de l'ADN est sensiblement modifiée, le petit sillon est élargi. Il est possible que le facteur $\mathrm{PBC}$, en modifiant légèrement la structure de l'ADN, facilite la liaison de la protéine HOX. La seule différence entre les deux analyses cristallographiques réside dans le fait que la région carboxy-terminale de la protéine $\mathrm{Pbx} 1$, c'est-à-dire les 13 résidus situés en aval de l'homéodomaine, a été conservée [3] ou non [2]. Cette région forme une quatrième hélice $\alpha$ qui se replie sur l'homéodomaine de $\mathrm{Pbx} 1$ et le contacte à la fois au niveau de l'hélice 1 et au niveau de l'hélice 3 . Ces résidus semblent ainsi jouer un rôle structural en stabilisant l'hélice 3 de l'homéodomaine contre l'ADN, ce qui corrobore les données biochimiques montrant que l'extension carboxy-terminale de Pbx1 augmente de 5 fois son affinité pour l'ADN [4]. Globalement, l'analyse cristallographique montre que les interactions protéine-protéine et les interactions protéine-ADN concourent à la formation du complexe ternaire.
Les protéines $\mathrm{PBC}$ ont la propriété de choisir leur partenaire d'hétérodimérisation en fonction de subtiles différences de la cible génomique et, notamment, en fonction de la base 6 du site de reconnaissance avec laquelle, pourtant, elles n'interagissent pas. De plus, les résidus composant le bras amino-terminal de l'homéodomaine des protéines HOX, qui, eux, interagissent avec la base 6 , jouent manifestement un rôle in vivo dans la spécificité de la protéine HOX alors que l'analyse cristallographique montre qu'ils n'entrent pas en contact avec la protéine PBC. Il est par conséquent improbable que le choix de la cible de l'hétérodimère PBC-HOX soit exclusivement déterminé par la protéine PBC. Cela suggère l'intervention d'autres co-facteurs. Un certain nombre de données génétiques et biochimiques vont également dans ce sens. Par exemple, chez la drosophile, le gène homéotique Deformed (Dfd) contrôle son propre taux d'expression par la fixation coopérative d'Exd et Dfd sur la séquence 5'TGATTAATGG-3' de son promoteur. In vivo, le remplacement du dinucléotide central TA par GG transforme le profil d'expression de $D f d$ en un profil d'expression de type labial, un autre gène Hox, ce qui sug- gère que ces deux nucléotides interviennent dans le choix du recrutement d'un hétérodimère Exd-Labial plutôt que d'un hétérodimère DfdExd [5]. Au contraire, in vitro, l'hétérodimère Exd-Labial se lie à la séquence 5'-TGATTAATGG-3'. D'autres partenaires protéiques interagissent donc vraisemblablement avec les complexes PBC-HOX/ADN. D'autres protéines à homéodomaine de type TALE, codées par le protooncogène murin Meis1, par homothorax (hth), l'orthologue de Meis1 chez la drosophile, par le gène murin Prep1 et par le gène de Caenorhabditis elegans ceh-25 interagissent avec les protéines PBC. La protéine Hth forme un hétérodimère avec Exd en l'absence d'ADN et cette interaction provoque la translocation d'Exd dans le noyau [6]. De cette façon, hth contrôle indirectement l'activité des protéines HOX. Inversement, les gènes Hox contrôlent le niveau d'Exd dans le noyau en agissant sur la transcription d'hth. Cela suggère que les rapports stœechiométriques des trois protéines dans le noyau sont finement réglés et jouent un rôle important. La fonction de translocation nucléaire d'une protéine PBC grâce à l'hétérodimérisation avec une protéine de la famille Meis n'a pas été décrite jusqu'à présent chez les mam- 
mifères. Néanmoins, on sait que Meis1 et Pbx1 peuvent former des hétérodimères au niveau de certaines séquences régulatrices, indépendamment des protéines HOX, par exemple au niveau de l'élément régulateur dépendant de l'AMPc du promoteur du gène bovin CYP17 [7]. Très récemment, la démonstration de la fixation de Meis1 au complexe ternaire HoxA9-Pbx2/ADN dans les cellules myéloïdes de souris [8] est venue étayer l'hypothèse d'une participation générale des protéines Meis/Hth aux complexes transcriptionnels PBC-HOX/ADN.

En conclusion, le rôle de facteur de transcription sélecteur joué par les protéines HOX est lié à la formation de complexes multimériques avec d'autres protéines, elles-mêmes à homéodomaine, qui par ailleurs coopèrent à la régulation de certains promoteurs. On peut finalement se demander qui, des protéines $\mathrm{PBC} / \mathrm{Meis} / \mathrm{Hth}$ ou des protéines HOX, sont les co-facteurs. I poos1 téines HOX n'apporteraient-elles pas seulement l'information de position nécessaire au bon fonctionnement des couples d'homéoprotéines déjà présents ?

\section{Remerciements}

À Jean Deutsch pour ses conseils avisés, à Aurore Lopez et Yves Maurin pour leur lecture attentive du manuscrit.

1. Gehring WJ, Affolter M, Bürglin T. Homeodomain proteins. Annu Rev Biochem 1994; 63: 487526.

2. Passner JM, Ryoo HD Shen L, Mann RS, Aggarwal AK. Structure of a DNA-bound UltrabithoraxExtradenticle homeodomain complex. Nature 1999; 397: 714-9.

3. Piper DE, Batchelor AH, Chang CP, Cleary ML, Wolberger C. Structure of a HoxB1-Pbx1 heterodimer bound to DNA: role of the hexapeptide and a fourth homeodomain helix in complex formation. Cell 1999; 96: 587-97.

4. Green NC, Rambaldi I, Teakles J, Featherstone MS. A conserved C-terminal domain in PBX Bंocruases DNA binding by the PBX homeodoE-mail : rbanissolpasteur.fr main and is not a primary site of contact for the YPWM motif of HOXA1. J Biol Chem 1998; 273: 13273-9.

5. Chan SK, Ryoo HD, Gould A, Krumlauf R, Mann RS. Switching the in vivo specificity of a minimal Hox-responsive element. Development 1997; 124: 2007-14.

6. Rieckhof GE, Casares F, Ryoo HD, Abu-Shaar M, Mann RS. Nuclear translocation of extradenticle requires homothorax, which encodes an extradenticle-related homeodomain protein. Cell 1997; 91: 171-83.

7. Bischof LJ, Kagawa N, Moskow JJ, et al. Members of the meisl and pbx homeodomain protein families cooperatively bind a cAMP-responsive sequence (CRS1) from bovine CYP17. J Biol Chem 1998; 273: 7941-8.

8. Shen WF, Rozenfeld S, Kwong A, Kömüves LG, Lawrence HJ, Largman C. HOXA9 forms triple complexes with PBX2 and MEIS1 in myeloid cells. Mol Cell Biol 1999; 19: 3051-61.

\section{Frédérique Peronnet}

UMR Cnrs 7622, Biologie moléculaire et cellulaire du développement, Université Pierre-et-Marie-Curie, 9, quai Saint-Bernard, 75252 Paris Cedex 05, France. 